\title{
Approaching the optimum focusing of diffused light through scattering media with iterative wavefront shaping by a probability-based algorithm
}

\section{Chi Man Woo}

The Hong Kong Polytechnic University https://orcid.org/0000-0003-1653-5029

\section{Qi Zhao}

The Hong Kong Polytechnic University

\section{Tianting Zhong}

The Hong Kong Polytechnic University

\section{Huanhao Li}

The Hong Kong Polytechnic University

\section{Zhipeng Yu}

The Hong Kong Polytechnic University

Puxiang Lai ( $\sim$ puxiang.lai@polyu.edu.hk)

The Hong Kong Polytechnic University

\section{Article}

Keywords:

Posted Date: January 18th, 2022

DOI: https://doi.org/10.21203/rs.3.rs-1198374/v1

License: (1) This work is licensed under a Creative Commons Attribution 4.0 International License. 


\title{
Approaching the optimum focusing of diffused light through scattering media with iterative wavefront shaping by a probability-based algorithm
}

\author{
Chi Man Woo ${ }^{1,2 \dagger}$, Qi Zhao ${ }^{1,2 \dagger}$, Tianting Zhong ${ }^{1,2}$, Huanhao Li ${ }^{1,2}$, Zhipeng \\ $\mathrm{Yu}^{1,2^{*}}$, Puxiang Lai ${ }^{1,2,3^{* *}}$ \\ ${ }^{1}$ Department of Biomedical Engineering, Hong Kong Polytechnic University, Hong Kong SAR \\ ${ }^{2}$ Hong Kong Polytechnic University Shenzhen Research Institute, Shenzhen, China \\ ${ }^{3}$ Photonics Research Institute Hong Kong Polytechnic University, Hong Kong SAR \\ ${ }^{*}$ Corresponding author: yu.zh.yu@polyu.edu.hk \\ ** Corresponding author: puxiang.lai@polyu.edu.hk \\ These authors contributed equally to this work
}

\begin{abstract}
Iterative wavefront shaping is a powerful tool to overcome medium scattering and enable focusing of diffusive light, which has exciting potentials in many applications that desire localized light delivery at depths in tissue-like complex media. Unsatisfactory performance and efficiency, however, have been a long-standing problem, and the large discrepancy between theoretical and experimental results has hindered the wide applications of the technology. Currently, most algorithms guiding the iterative search of optimal phase compensation rely heavily on randomness to achieve solution diversity. The lack of clear guidance on the new solution generation process considerably affects the optimization efficiency. Therefore, we propose a probability-based iterative algorithm that the new solutions are generated based on a probability map. With the clearer guidance provided by the probability map and less involvement of randomness, we can obtain optimization results that efficiently approach the theoretical optimal value. Moreover, with the proposed algorithm, we demonstrate higher adaptability in an unstable scattering environment and more spatially uniform optical focusing in the field of view. This study advances the state of the art in the practice of iterative wavefront shaping and can potentially inspire or open up wide applications that desire localized and enhanced optical delivery in situ.
\end{abstract}

\section{Introduction}

High-resolution optical imaging techniques such as fluorescence microscopy, optical coherence tomography, and photoacoustic imaging have become increasingly important in biomedicine in recent decades. These imaging modalities mostly rely on ballistic/quasi-ballistic photons or the photoacoustic effect to provide optical contrast of the target region. However, due to the inhomogeneities of refractive index in biological tissue, photons are multiply scattered in tissue sample and the amount of ballistic photons decays exponentially with increasing propagation depth, limiting high-resolution optical imaging to a depth of $\sim 1 \mathrm{~mm}$ beneath the skin or tissue surface ${ }^{1}$. Thanks to the emergence of optical wavefront shaping techniques, scattering-induced wavefront distortions nowadays can be compensated via various approaches, such as optical phase conjugation ${ }^{2-8}$, transmission matrix method ${ }^{9-12}$, iterative optimization ${ }^{13-29}$, and artificialintelligence-assisted methods ${ }^{30-33}$, allowing optical focusing or imaging inside/through scattering media. Iterative optimization approaches are widely adopted because they are straightforward and less technically demanding. In this technique, the wavefront of incident light is iteratively modulated by a spatial light modulator (SLM) according to the feedback signals from a detector (e.g., camera or photodetector) placed behind the scattering medium or a guidestar (e.g., fluorescent or photoacoustic emission as a virtual guidestar ${ }^{34,35}$ ) within the 
scattering medium, so that the optical distortions can be compensated and an optical focus or a desired output field can be obtained at the target location. Several metaheuristic optimization algorithms, such as genetic algorithm (GA) ${ }^{15-18}$, particle swarm optimization (PSO) ${ }^{19-23}$, simulated annealing algorithm (SA) ${ }^{24,25}$, bat algorithm (BA) ${ }^{28}$, and separable natural evolution strategies (SNES) ${ }^{29}$, have been demonstrated for successful optical focusing inside/through scattering media. Aiming for faster and better optimization results, researchers have improved different algorithms primarily in three ways: optimizing parameters, introducing variants of the algorithms (e.g., microgenetic algorithm $(\mu \mathrm{GA}){ }^{17}$ and particle swarm optimization with mutation ${ }^{23}$ ), and exploiting hybrid algorithms (e.g., parameter-free algorithm (PFA), a combination of genetic algorithm and bat algorithm ${ }^{27}$, and Genetic Neural Network (GeneNN), a hybrid of GA and deep neural networks ${ }^{31}$ ).

Most of these metaheuristic algorithms are stochastic (i.e., non-deterministic), that is, the new solution generating process involves a lot of randomness. For example, in GA, the parents are randomly selected from the population and the new offspring randomly inherits the genes from either its father or mother (i.e., random crossover); in PSO, random numbers play a role in computing the new velocities and positions of the particles. Randomness helps to enhance the diversity of potential solutions and prevent the optimization from falling into local optima. Nevertheless, it does not always lead to the formation of good solutions. For instance, in GA, the better performing individuals may not be selected as parents, and even so, good parents do not necessarily breed good solutions, since the good genes may not have been passed on to the offspring. Although there are a set of equations and rules guiding the optimization to gradually improve the solutions, the mechanism of how the algorithms produce a good solution is not yet clear, as they depend heavily on randomness. The lack of clear guidance for the process of generating new solutions may affect the optimization efficiency, and there is no guarantee that a global optimum will be found. This could be one of the reasons why most of the optical focusing results by iterative approaches are far from the theoretical optimum, which, together with the unsatisfactory focusing speed, have hindered the applications of iterative wavefront shaping to biological tissue or other unstable scattering materials. As each of existing algorithms has its strengths and limitations, there is no iterative algorithm that can surmount the abovementioned obstacles. It is challenging for us to achieve nearly perfect performance with a single algorithm.

In this study, we propose a hybrid algorithm that integrates GA and ant colony optimization (ACO) for optical focusing inside/through scattering media, and aims to exploit the advantages and overcome the limitations of the two algorithms. GA has superior resistance to noise but relatively slow convergence; ACO, on the other hand, can search for good solutions quickly but is prone to local convergence ${ }^{36}$. The hybrid algorithm, termed as GA-ACO, has the basic structure of GA, with the genetic operations (i.e., parent selection and crossover) replaced by the pheromone update procedures of ACO. Thus, ACO helps to generate good solutions quickly, and the mutation process of GA helps to prevent premature convergence. Unlike other metaheuristic algorithms, new solutions in GA-ACO are generated according to a probability map and hence less randomness is involved in the process, which ensures the optimization performance and efficiency. The hypothesis is computationally shown that GA-ACO can effectively search for the global optimum and experimentally demonstrated that it can achieve a final peak-to-background ratio (PBR) of optical intensity approaching to the theoretical enhancement value in binary-amplitude modulation.

\section{Results}

\section{Principle of GA-ACO}


Genetic algorithm has been one of the most popular iterative optimization algorithms for wavefront shaping since Vellekoop and Mosk first proposed its usage ${ }^{13}$ and Conkey first demonstrated successful wavefront control through highly scattering media ${ }^{15}$. It is an evolutionary algorithm inspired by the natural selection process in biological systems. It starts with a population of potential solutions and the solutions are ranked by their fitness (i.e., how well they fit the optimization problem). Individuals with higher fitness have a higher probability of being selected as parents to generate new solutions. The crossover rate is usually $50 \%$, which means that half of the elements of a new individual are inherited from its mother and the other half are from its father, and mutation takes place to change the value of some randomly selected elements. These processes of selection, crossover, and mutation produce generations of offspring and eliminate old individuals with lower fitness. As a result, the solutions gradually evolve towards the global optimum.

Ant colony optimization is a probability-based metaheuristic optimization algorithm often used in routing or travelling salesman problems, for example, finding the shortest path to travel through a series of cities ${ }^{37,38}$. The idea of ACO comes from the foraging behavior of ants in a colony, as illustrated in Fig. 1a. In a simplest form, there are two paths that the ants can take to reach the food, but the distances are unknown. Initially, the ants wander randomly along different paths to search for food and return to their colony after finding the food. Along the way, the ants lay down pheromones to communicate with other ants as they walk back and forth between their colony and the food source. The shorter the path the ants choose, the faster they commute, and the more pheromones accumulate along the way, thus attracting more ants to choose that path, resulting in a positive feedback loop. In the end, the entire ant colony travels with the shortest path.

ACO's greatest strengths lies in its ability to quickly search for good solutions and effectively adapt to dynamic changes ${ }^{36}$, which ideally meets the requirements for efficiency and adaptability in wavefront shaping in nonstationary scattering media. Although ACO is usually applied to routing problems, the concept can also fit into wavefront shaping for optical focusing inside/through scattering media with modest modifications. The following elucidation is based on binary-amplitude wavefront modulation with a digital micromirror device (DMD) to have a higher refreshing rate than phase modulation with a liquid crystal-based SLM. Similar to GA, ACO starts with a population of potential solutions (i.e., the DMD mask), which can be referred to "ants" here, and aims to find a DMD mask that brings about the strongest optical focusing effect. Every independently controlled element or block on the DMD mask can be treated as a city: if the element is "ON", ants will pass through the corresponding city (status "1"); if the element is "OFF", ants will not pass through the corresponding city (status " 0 "). The fitness of each DMD mask, for example, peak-to-background ratio (PBR) or peak focal intensity, is measured to represent the amount of pheromone deposited by a single ant in each status in each city.

$$
\Delta \tau_{L}^{m, i}=\text { fitness },
$$

where $\Delta \tau_{L}^{m, i}$ denotes the pheromone deposited by the $m^{t h}$ ant at the $i^{t h}$ pixel on status $L$ (either 0 or 1). Then, the pheromone data from each ant are gathered to form a pheromone accumulation in each $\operatorname{city}\left(\tau_{L}^{i}\right)$.

$$
\tau_{L}^{i}=\sum_{m=1}^{M} \Delta \tau_{L}^{m, i},
$$

where $M$ is the total number of ants in the population. Eq. (2) can be used to calculate the total amount of pheromones in each status in different cities. At last, a probability map $\left(p_{L}^{i}\right)$ can be constructed showing whether the "0" or "1" status is more likely to produce a better result in each city.

$$
p_{L}^{i}=\tau_{L}^{i} / \sum_{L} \tau_{L}^{i},
$$


The probability map is then used as an indicator to build the next generation of potential solutions. These processes of computing the pheromone accumulation and the probability map are repeated until a global optimum is found. Nevertheless, in the real case, it is prone to fall into local optima instead. Despite the fact that the process of generating a new solution does not involve randomness, which can help to search for good solutions rapidly, the population gradually loses diversity, causing the optimization to be trapped in a local optimum. Therefore, ACO alone is not an ideal algorithm for wavefront shaping ${ }^{39}$.

In view of this, to take the advantages and overcome the limits of both GA and ACO, a hybrid algorithm, GA-ACO, is proposed. The flowchart of the hybrid algorithm is shown in Fig. 1b. As seen, GA-ACO has the basic structure of GA, where the crossover process has been replaced by the pheromone matrix and the probability map update process. First, a set of DMD masks is randomly generated as the initial population and the fitness of each mask is measured. Then, ACO is responsible for generating the offspring. The pheromone value of each path for each DMD element is calculated according to Eqs. (1) and (2), and a probability map is computed according to Eq. (3) to show the probability of each DMD element being "ON" or "OFF", thus guiding the process for generating new offspring. After that, mutation of the offspring is performed to enhance the solution diversity. In this process, a portion of the elements is randomly selected, and they are flipped either from "ON" to "OFF" or from "OFF" to "ON". The fitness of all the newly generated individuals is evaluated and the old solutions with lower fitness are replaced. The process is repeated until a termination condition is satisfied or a global optimum is found.

a

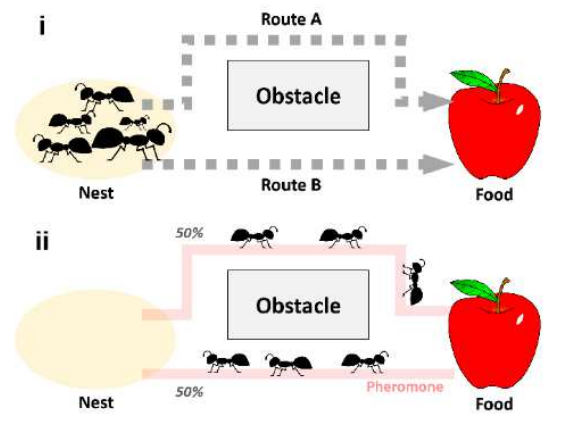

iii

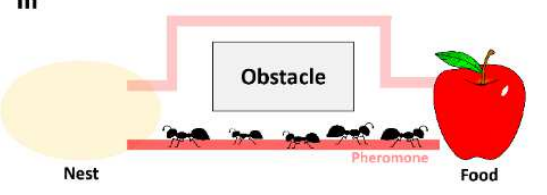

b

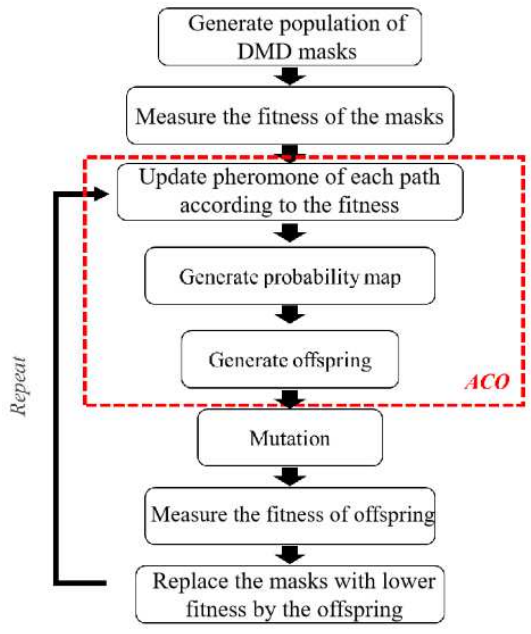

Fig. 1. Principle of GA-ACO. (a) Concept of ant colony optimization. i) The ants can travel along route $\mathrm{A}$ or route $\mathrm{B}$ to reach the food. ii)The ants wander randomly along different paths to search for food and return to their colony. They lay down pheromones to communicate with one another. iii) The entire ant colony travels with the shortest path. (b) Flow chart of GA-ACO.

\section{Theoretical enhancement value}

The peak-to-background ratio (PBR) is commonly used as a quantitative index to evaluate the performance of wavefront shaping results. It is defined as the ratio between the average focal intensity and the average background intensity. In a strong scattering regime, the theoretical enhancement value that can be achieved is equal to ${ }^{40}$

$$
\eta=\alpha(N-1)+1
$$


where $N$ is the number of control units on the SLM (i.e., the input modes of the modulation) and $\alpha$ is the relative enhancement value determined by the type of the modulation, for instance, phase modulation or amplitude modulation. As we focus on binary-amplitude modulation in this study, $\alpha$ is equal to $1 / 2 \pi$. Therefore, the theoretical enhancement value in the context is $\frac{1}{2 \pi}(N-1)+1^{41}$.

\section{Numerical verification}

Numerical simulations are performed to demonstrate the effectiveness and efficiency of GA$\mathrm{ACO}$ and to verify that the algorithm is capable of achieving a global optimum. First, a transmission matrix $(\mathrm{T})$ is created to mimic the scattering phenomenon when light passes through a scattering medium. The transmission matrix $(T)$ relates the input field $\left(E_{i n}\right)$ and the output field $\left(E_{\text {out }}\right)$ in the following linear relationship:

$$
E_{\text {out }}=T E_{\text {in }} .
$$

In our simulation, the input is a 1 -bit vector (i.e., either " 0 " or " 1 ") of size $1024 \times 1$ and the output is a complex vector of size $1 \times 90,000$, so T contains $90,000 \times 1024$ elements $\left(t_{m n}\right)$, which individually relates the $\mathrm{n}^{\text {th }}$ channel of $E_{\text {in }}$ to the $\mathrm{m}^{\text {th }}$ channel of $E_{\text {out }}$. To maximize the intensity of a focal point at the target location, the input elements that contribute positively to the focus should be switched on and those that contribute negatively should be switched off. That is,

$$
\text { input channel }=\left\{\begin{array}{l}
1, \operatorname{Re}\left(t_{m n}\right)>0 \\
0, \text { otherwise }
\end{array} .\right.
$$

Therefore, from the simulated transmission matrix, an ideal DMD mask (Fig. 2c(i)) that leads to the highest focusing intensity can be easily predicted, which is considered here as the theoretical optimal pattern. It can be interpreted that if we can obtain DMD patterns that are highly similar to the optimal one, we can achieve optimization results that are close to the global optimum.

Iterative optimizations by GA and GA-ACO are then carried out based on the same transmission matrix. Both GA and GA-ACO start from a randomly generated population of DMD masks, and the parameters used by the two algorithms are exactly the same (Table. 1), where the population size is 50 and the offspring size is 25 . An exponentially decaying mutation regime is adopted, with the initial mutation rate, final mutation rate, and decay constant set to $0.008,0.001$, and 250 , respectively. The intensity of the target position is used as a feedback signal/fitness function to evaluate the performance of the masks in the simulation. Every time a feedback signal is measured, it is counted as one measurement. In GA and GA-ACO, one iteration is completed when the fitness values of all offspring are recorded. After 12,500 measurements (500 iterations), the optimized patterns from GA and GA-ACO are compared with the theoretical optimal pattern. It can be observed that the resulting pattern from GA-ACO (Fig. 2d(i)) is about $97 \%$ similar to the theoretical optimal pattern (Fig. 2c(i)). In other words, only around 30 out of 1024 elements of the resulting pattern differ from the optimal pattern. The final intensity of the target position (Fig. 2d(ii)) is also comparable to that of the theoretical optimal output (Fig. 2c(ii)). Meanwhile, for GA, the similarity between the resulting pattern (Fig. 2b(i)) and the theoretical optimal pattern (Fig. 2c(i)) is around 92\%, resulting in a relatively lower final peak intensity (Fig. 2b(ii)). The simulation is repeated for 30 times for each algorithm. The distribution of the results is shown in Fig. 2e and the curves in Fig. $2 \mathrm{f}$ are the averaged results. It is noteworthy that GA-ACO has a higher similarity to the theoretical optimal pattern than GA in all trials. The highest similarity achieved by GA-ACO is over $98 \%$ and the lowest is $96 \%$. On the other hand, for GA, the similarity ranges from $89 \%$ to $93 \%$. In addition to the higher similarity, GA-ACO also demonstrates higher efficiency in finding good solutions as only 6625 measurements are required to achieve $92 \%$ similarity. The simulation 
results suggest that with a probability-based solution generation process from ACO, optimization results approaching the global optimum can be achieved with high efficiency, although the difference from the simulation results obtained with GA is not that striking.

Table. 1 Initial paraemters for GA and GA-ACO

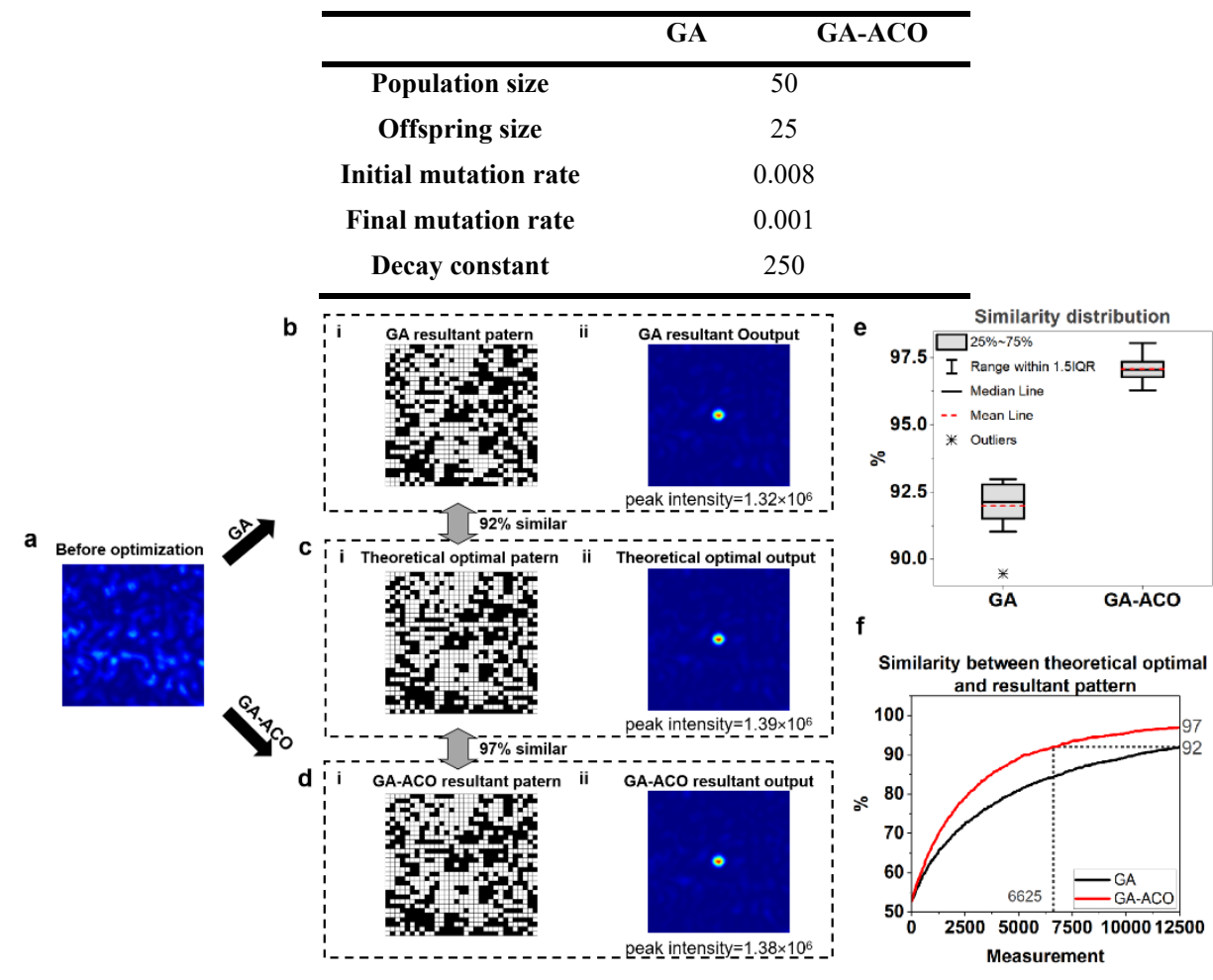

Fig. 2. Numerical simulation results. (a) Speckle pattern before optimization. Optimization result from (b) GA, (c)theoretical prediction, and (d) GA-ACO with i) output DMD patterns and ii) corresponding output light fields. (e) Distribution of the data from 30 trials. (f) Similarity growth during the optimization.

\section{Single-focus performance of GA and GA-ACO}

Various experiments have been conducted to assess the focusing ability and efficiency of GAACO with the experimental setup shown in Fig. 3. Ground glass and multimode fiber are used as the scattering media in different sets of experiments. The ground glass creates a highly stable scattering environment while the multimode fiber mimics an unstable medium with a fast decorrelating scattering environment. The performance of GA-ACO is fairly compared with the traditional GA under the condition that they share the same set of parameters as listed in Table. 1, and the only difference between them is the new solution generation procedure. Therefore, we can demonstrate the effectiveness of introducing a probability-based regime to reduce the involvement of randomness. PBR is used as the feedback signal in the experiments and each PBR measurement takes around 25 milliseconds. 


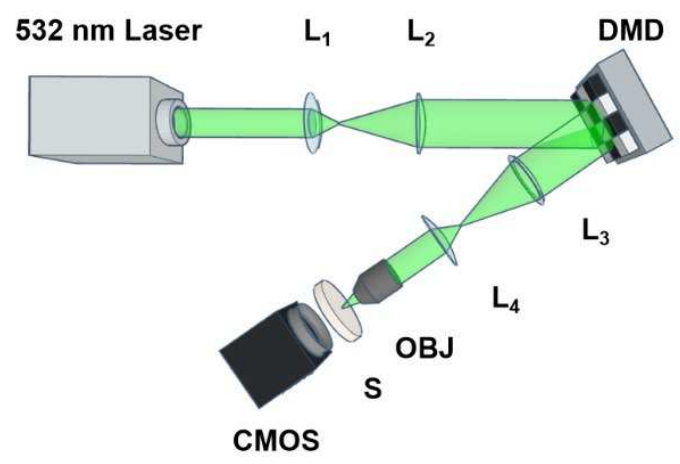

Fig. 3. Experimental setup. L1-4: Convex lenses; DMD: digital micromirror device; OBJ: 25x objective lens; S: scattering medium (ground glass and multimode fiber in this study).

First, to compare the efficiency of GA and GA-ACO, different scattering media and different numbers of input modes were used in experiment. The optimization efficiency herein is evaluated based on the PBR enhancing speed and the ratio between the maximum PBR achieved and the theoretical optimum. Fig. $4 \mathrm{a} \& \mathrm{~b}$ show the images captured by the camera behind the ground glass and multimode fiber with $32 \times 32$ input modes respectively. Using ground glass as the scattering medium, as shown in Fig. 4a(i), the initial PBR of the target location was 3.2. The optimization results by GA and GA-ACO are shown in Fig. 4a(ii) and (iii), respectively. According to Eq. (4), as $\mathrm{N}$ is equal to $32 \times 32$ and $\alpha$ is equal to $1 / 2 \pi$ for binaryamplitude modulation, the theoretical enhancement value is 163.8. The maximum PBR of the focal point obtained by GA was 88.9 , which is $54 \%$ of the theoretical value; with GA-ACO, the PBR of the focal point was increased to 159.5 , which is $97 \%$ of the theoretical value. The PBR growth during the optimizations by GA and GA-ACO is shown in Fig. 4c. The blue dashed horizontal lines in the figures indicate the theoretical enhancement value, and the black and red solid lines respectively represent the results from GA and GA-ACO. As seen, GA and GAACO reach their highest values $\left(\operatorname{Max}_{\mathrm{GA}}\right.$ and $\left.\mathrm{Max}_{\mathrm{GA}-\mathrm{ACO}}\right)$ at the $9125^{\text {th }}$ and $12300^{\text {th }}$ measurements, respectively, and $\mathrm{Max}_{\mathrm{GA}-\mathrm{ACO}}$ is close to the theoretical value (163.8). However, GA began to plateau after around 5000 measurements and appears to have converged to a local optimum.

When a multimode fiber was used as the scattering medium, the initial PBR of the target point was 8.3 (Fig. 4b(i)). The maximum PBR of the focal point obtained by GA (Fig. 4b(ii)) and GA-ACO (Fig. 4b(iii)) was 65.3 (40\% of the theoretical value) and $111.6(68 \%$ of the theoretical value), respectively. As can be seen from the PBR growth curve in Fig. 4d, GA reached its highest values at the $5680^{\text {th }}$ measurement. But after that, the PBR started to decrease gradually and the final PBR became 53.6 after 12500 measurements, which has experienced a

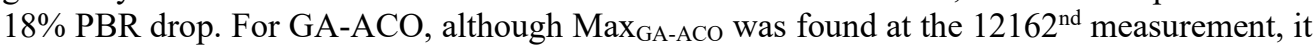
seems that the PBR still had the chance to grow.

The PBR drop in GA with multimode fiber as the scattering medium could be attributed to the instability of the scattering environment. Fig. 4e depicts the system stability during the experiments with ground glass and multimode fiber. The stability is evaluated by observing the change of the output speckle patterns over time with the same input wavefront. The stability test was repeated 5 times for each condition and the results are averaged. It can be observed that when ground glass was used as the scattering medium, the Pearson's correlation coefficient (c.c.) between the speckle pattern at the beginning and that during the measurement remained to be close to 1 , which means that the system was highly stable and the optimizations were performed in a low-noise situation. However, when a multimode fiber was used as the scattering medium, the correlation coefficient gradually decreased from 1 to around 0.83 after 12500 
measurements (around 5.2 minutes), indicating that the speckle patterns were rapidly decorrelated. The optimization algorithm should have high efficiency to keep up with the dynamic changes of the transmission matrix ${ }^{40}$. Therefore, the experimental results suggest that GA-ACO has a higher efficiency than GA, and GA-ACO can better adapt to the decorrelation effect.

In addition to the $32 \times 32$ setting employed with $14 \times 28$ DMD pixels grouped as a superpixel to modulate the wavefront, the DMD was also divided into $16 \times 16$ and $64 \times 64$ segments, with $28 \times 56$ and $7 \times 14$ DMD pixels grouped as a superpixel, respectively. The total number of DMD pixels used is the same in all 3 cases. The experiments were performed with ground glass as the scattering medium. With $16 \times 16$ input modes (Fig. 4f), both GA and GA-ACO can achieve PBR not far away from the theoretical value (41.6). GA reached its maximum at the $4822^{\text {nd }}$ measurement, while GA-ACO topped out at the $3001^{\text {st }}$ measurement. It can be observed that GA-ACO needed only 1213 measurements to attain the same level of Max $_{\mathrm{GA}}$, which is almost 4 times faster than GA. With $64 \times 64$ input modes (Fig. 4g), the difference in focusing performance between GA and GA-ACO becomes more apparent. The highest PBR reached by GA in 37500 measurements is 171.9 , while GA-ACO reached a similar level of at the $5464^{\text {th }}$ measurement and hit its maximum of 488.1 at the $31375^{\text {th }}$ measurement. In all 3 cases, GAACO demonstrates a higher PBR enhancing efficiency and more promising focusing ability than GA does.

The $\operatorname{Max}_{\mathrm{GA}}$ and $\mathrm{Max}_{\mathrm{GA}-\mathrm{ACO}}$ for each case are summarized in Table. 2. They are compared with the theoretical enhancement value, and the results are illustrated in Fig. 4h. It is noticeable that the experimental enhancement percentage to the theoretical value achieved for both GA and GA-ACO decreases as the number of input modes increases. It becomes more difficult to approach the theoretical optimum for a large number of input modes as the number of possible solutions increases exponentially. More measurements are also needed for the optimization to converge, causing it to suffer more from noise. Furthermore, the decreasing percentage can also be attributed to the diffraction effect of the DMD grating. The more segments the DMD is divided, the more the results are affected by diffraction. With $16 \times 16$ and $32 \times 32$ input modes, GA-ACO is found to have optimization results very close to the theoretical values, which is, to the best of our knowledge, unprecedented optimization efficiency in wavefront shaping obtained in experiment. With $64 \times 64$ input modes, it can still achieve $75 \%$ of the theoretical value. Although GA can achieve PBR approaching the theoretical value with the $16 \times 16$ input modes, the percentage is drastically reduced when a larger number of input modes are used.

Table. 2 Maximum PBR achieved different conditions

\begin{tabular}{cccc}
\hline \multirow{2}{*}{ No. of input modes } & \multirow{2}{*}{ Theoretical value } & \multicolumn{2}{c}{ Max PBR achieved } \\
\cline { 3 - 4 } & & GA & GA-ACO \\
\hline $\mathbf{1 6} \times \mathbf{1 6}$ & 41.6 & $36.4(\sim 88 \%)$ & $43.9(\sim 106 \%)$ \\
$\mathbf{3 2} \times \mathbf{3 2}$ & 163.8 & $88.9(\sim 54 \%)$ & $159.6(\sim 97 \%)$ \\
$\mathbf{6 4} \times \mathbf{6 4}$ & 652.7 & $171.9(\sim 26 \%)$ & $488.1(\sim 75 \%)$ \\
\hline
\end{tabular}


a

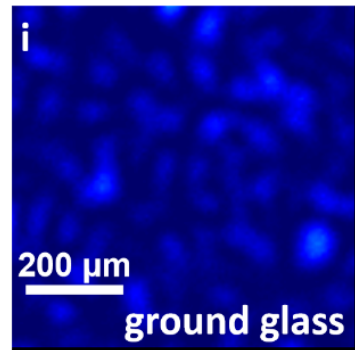

b i

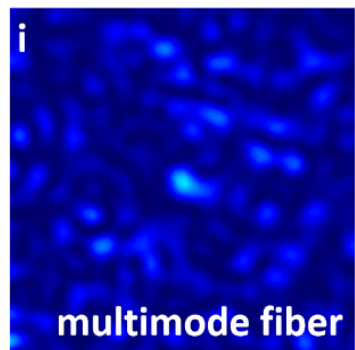

C

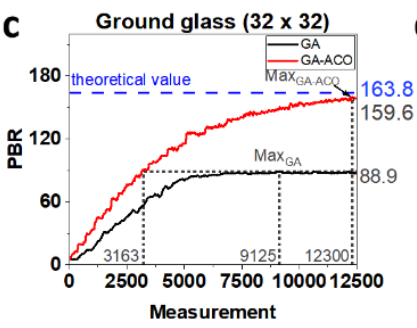

f

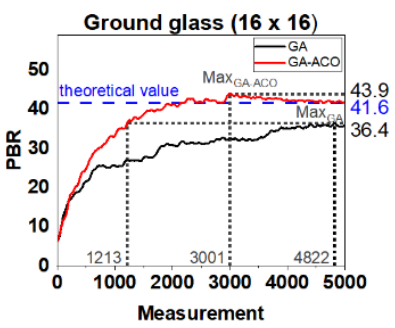

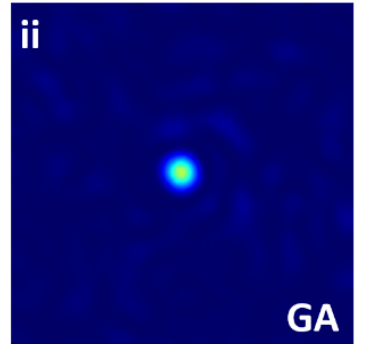
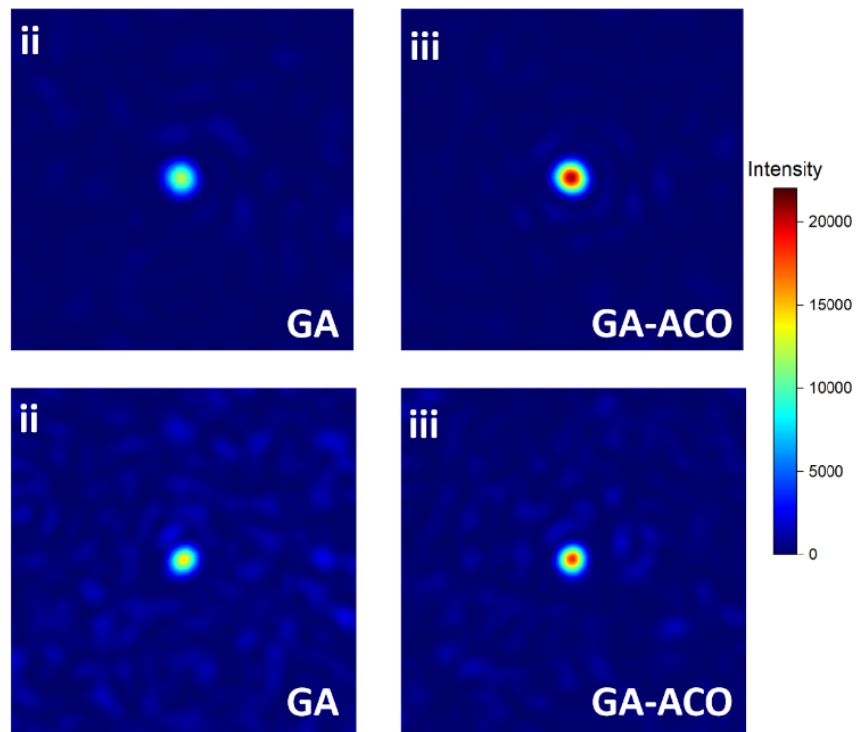

d 150 Multimode fiber $(32 \times 32)$

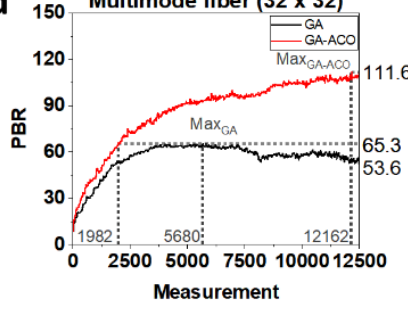

g

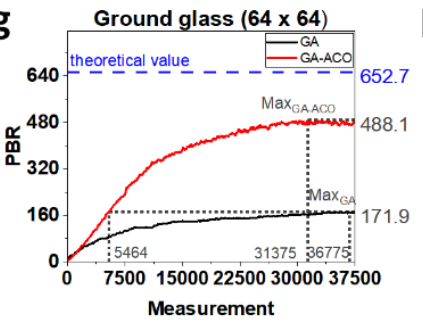

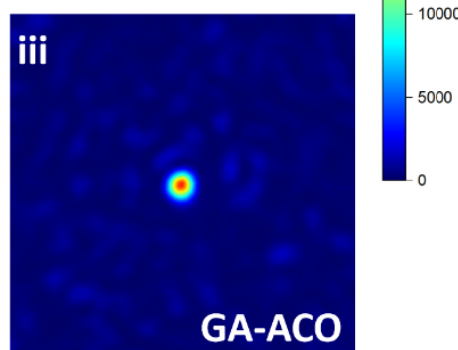

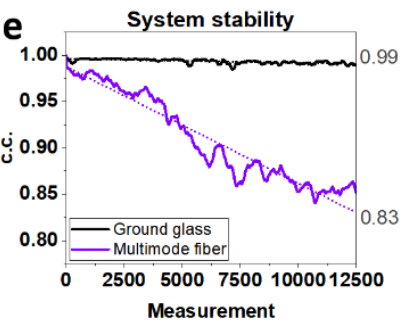

h

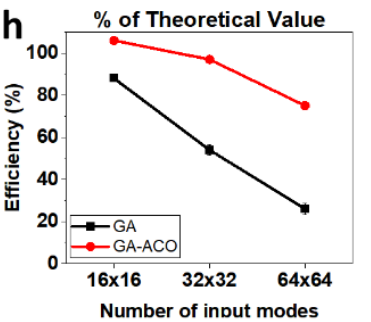

Fig. 4. Single-point focusing results under different conditions. Output patterns captured when (a) ground glass and (b) multimode fiber were used as the scattering medium with $32 \times 32$ input modes (i) before optimization, (ii) after GA, and (iii) after GA-ACO. The $200 \mu \mathrm{m}$ scale bar is applicable to (a) \& (b). (c \& d) PBR growth during optimization with ground glass and multimode fiber, respectively, as the scattering medium. Black and red solid lines represent the optimization results from GA and GA-ACO, respectively. Blue dashed horizontal lines represent the theoretical enhancement value under each condition. (e) System stability during the optimization with ground glass (black solid line) and multimode fiber (blue solid line) as the scattering medium. (f) PBR growth with $16 \times 16$ input modes. (g) PBR growth with $64 \times 64$ input modes. (h) Percentage of theoretical value achieved by GA (black solid line with square markers) and GA-ACO (red solid line with square markers) with different numbers of input modes.

\section{Multi- focus performance}

Next, the multi-point focusing ability of the algorithms was also investigated. Five points in the region of interest were selected for focusing, as illustrated in Fig. 5a. To ensure the intensity homogeneity of the foci, the following fitness function was adopted ${ }^{42}$ : 


$$
\text { fitness }=\sum_{k=1}^{K} I_{k}-K \cdot \sigma(I)
$$

where $K$ is the number of target points, $I_{k}$ is the intensity of the kth focal point, and $\sigma(I)$ is the standard deviation of the intensity of all the points. The theoretical enhancement value of each focus then becomes $\eta / K .32 \times 32$ input modes were used in this set of optimizations. The results by GA and GA-ACO are shown in Fig. 5a and b, respectively. It can be clearly seen that the 5 focal points from GA-ACO are brighter than those from GA. Fig. $5 \mathrm{c}$ also shows the growth of the average PBR of the focal points during the optimizations. The results are consistent with those from single-point focusing that GA-ACO can achieve PBR approaching the theoretical optimum and has a higher optimization efficiency.

a

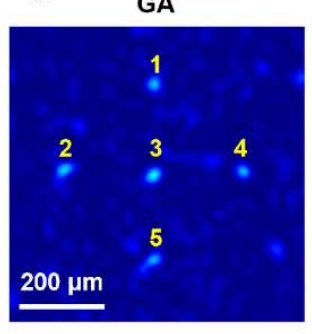

b

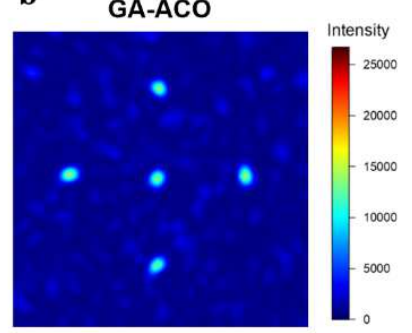

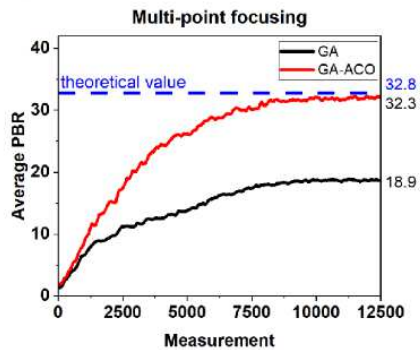

Fig. 5. Experimental results of multi-point focusing. 5 focal points obtained by (a) GA and (b) GA-ACO. The $200 \mu \mathrm{m}$ scale bar and the color bar are applicable to (a) and (b). (c) Average PBR growth during the optimization. Black solid line and red solid line represent the optimization results from GA and GA-ACO, respectively. Blue dashed horizontal lines represent the theoretical optimal average PBR.

\section{Focusing performance in different regions}

Last but not least, to investigate how the initial brightness of the target point affects the optimization result, eight different points in the region of interest with different initial intensity were selected for optimization. Fig. 6a shows the speckle field before optimization and the positions of the eight target points. As seen, some points are darker and some are brighter, and the eight points are numbered in ascending order of initial PBR. Then, the wavefront was modulated to produce focal points at these eight positions sequentially; at each position, both GA and GA-ACO were applied. Similar to the experiments mentioned above, $32 \times 32$ input modes were used, and each experiment took 12500 measurements. Fig. $6 \mathrm{~b}$ and $\mathrm{c}$ show the images of focal points obtained by the two algorithms, and the final PBR distribution of the eight points is shown in a boxplot in Fig. 6d.

On the whole, it can be noticed that the focal points from GA-ACO are much brighter than those from GA, regardless of the initial PBR. The mean value of the final PBRs from GA-ACO is 160.8 , which is almost twice of that from GA and is $\sim 98 \%$ of the theoretical enhancement value under the experiment condition. On the other hand, as seen from the GA optimization results (Fig. 6b), a point with a lower initial PBR, for example Point 1 and Point 2, generally leads to a lower final PBR, and vice versa for initially brighter positions. The final PBR ranges from 60.4 to 96.9 and has a standard deviation of 13.5. In contrast, with GA-ACO (Fig. 6c), the results are less spread out, with a standard deviation of only 4.7. Since the optimizations for all eight points approach the theoretical optimum, the room for improvement is limited, especially for the points with higher initial brightness. This could be the reason why the deviation between the final PBR from GA-ACO is much smaller than that from GA. It could also imply that the optimization result from GA-ACO is less likely to be affected by the initial brightness of the 
point. Even if a dark position is chosen, a good focusing result can still be obtained, which allows more flexibility in selecting the target point. This improvement of spatial uniformity for focuses is also practically impactful for laser scanning imaging and stimulation applications ${ }^{43}$.

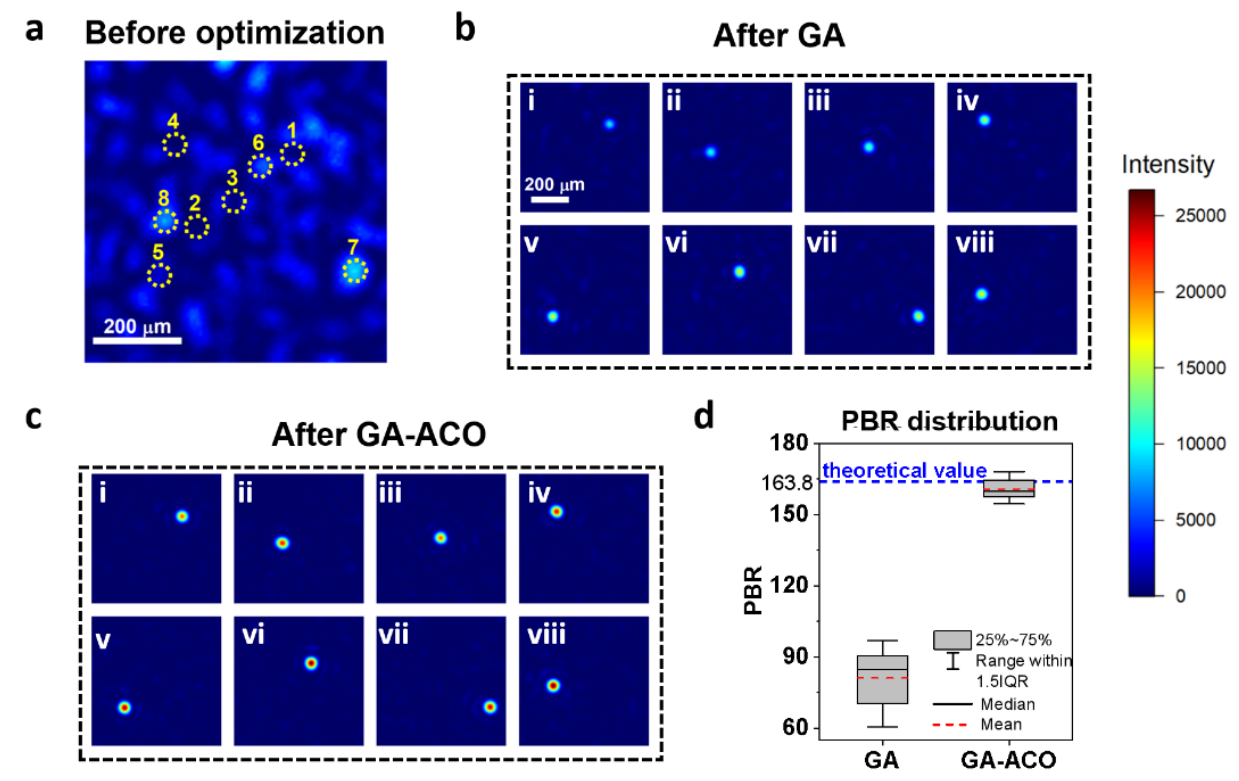

Fig. 6. Experimental results of focusing with different initial brightness. (a) Speckle pattern before optimization and the positions of the eight target points. (b) Resulting focal points obtained by GA. (c) Resulting focal points obtained by GA-ACO. (d) Distribution of PBR obtained from the eight focal points. The $200 \mu \mathrm{m}$ scale bar and the color bar are applicable to all images in (b) and (c).

\section{Discussion}

As seen from the simulation and experimental results, GA-ACO demonstrates excellent performance and efficiency in optical focusing through a scattering medium. GA-ACO can efficiently achieve optimization results approaching the theoretical optimum, and significantly outperforms the traditional GA. The only difference between GA and GA-ACO is the new solution generation process, which may imply that the probability-based approach is more effective than the parent selection and crossover processes in finding good solutions. With a probability map stating that a single pixel on the DMD should be "ON" or "OFF" to have a higher chance of contributing positively to the focus, GA-ACO can generate a DMD pattern that matches well with the transmission matrix, as evidenced by the simulation results. On the other hand, the efficiency of the optimization can be significantly increased as less time has to be spent on dealing with the unsatisfactory solutions generated by those processes with a lot of randomness.

GA is well-known for its superior noise-resistance ability ${ }^{44}$, yet GA-ACO can go above and beyond. As an evolution-based algorithm, GA relies heavily on the experience accumulated from generation to generation, but rarely considers the current state of the scattering medium ${ }^{26}$. The medium gradually decorrelates during the experiment, and when the decorrelation is large, the experience from the previous iterations may no longer be well-suited for the current generation, which affects the optimization efficiency and the result. This is one of the reasons why there are always discrepancies between the ideal and experimental results. Sometimes, it can also cause the optimization to be trapped in local optima. In contrast, GA-ACO does not 
rely so much on the results from previous iterations. The probability map is built independently according to the performance of the current generation based on the instant medium status. Therefore, it is easier for GA-ACO to adapt to medium decorrelation or environmental change, and to reduce the gap between the theoretical and practical results. Our experimental results have suggested that GA-ACO has a stronger noise-resistance ability and adaptability.

One may notice that some of the optimization results from our experiment exceed the theoretical enhancement values. Indeed, the aforementioned theoretical value was derived based on the focal intensity enhancement. As the transmission matrix follows a circular Gaussian distribution ${ }^{45}$, if we comply with the criterion that the DMD pixel is "ON" when $\operatorname{Re}\left(t_{\mathrm{mn}}\right)>0$ to maximize the intensity of the target point, half of the pixels on the optimal DMD pattern should be "ON", so the relative enhancement value $(\alpha)$ is taken as $1 / 2 \pi$. However, in this case, the "ON" pixels that contribute to the focus may also contribute to the background intensity. If we aim for a high PBR, only the pixels that contribute exclusively to the focus intensity but not the background intensity should be "ON". Chandrasekaran et al. proved that $\alpha$ for binary-amplitude modulation can be as large as 0.2 if a threshold is set to turn some "ON" pixels with low contribution to be "OFF" ${ }^{46}$, resulting in a larger maximum achievable PBR. However, for simplicity, in this study, we just compare our results with the conventional theoretical value. Chandrasekaran et al. also found that around $27 \%$ of the input modes should be "ON" to achieve the maximum PBR. In our experiment, the optimal DMD patterns obtained by GA and GA-ACO in the section "Focusing performance in different regions" have an average of $48 \%$ and $43 \%$ of "ON" pixels, respectively. This could be another reason why GA$\mathrm{ACO}$ has a higher PBR as more pixels contributing to the background intensity are "OFF".

The remarkably improved focusing ability and efficiency by GA-ACO could open up more application opportunities for iterative wavefront shaping in different fields. For example, it could be useful for photodynamic therapy (PDT), which is a treatment that use light to activate photosensitizing drugs to destroy nearby abnormal cells. However, the application of PDT is usually limited to superficial tissue. With a highly efficient optimization algorithm, combined with a high-speed wavefront shaping system, guide-star assisted deep-tissue PDT may become possible. Moreover, a higher PBR means that more energy is concentrated at the target position, thus less incident laser energy is required to activate the drugs and the risk of burning the surrounding tissue can be reduced. This study may also be useful for other non-biomedical applications, for example, optical communication with multimode fibers or in scattering environments such as haze or fog. With the highly efficient optimization algorithm, optical signal (e.g., quantum key distribution (QKD) ${ }^{47}$ and orbital angular momentum (OAM) ${ }^{48}$ ) transmission efficiency and accuracy through complex system can be enhanced.

It should be noted that although a significant improvement in optimization efficiency has been demonstrated in this study, the focusing speed of the proposed iterative algorithm is still not comparable to that of in-iterative wavefront shaping approaches, such as optical phase conjugation ${ }^{8}$ and transmission matrix measurement ${ }^{12}$. The optimization speed is still the main challenge in iterative wavefront shaping for wide applications, since patterns have to be computed and loaded onto the SLM at every iteration. A few hundred or even more iterations are usually needed for a good result, which inevitably makes the time required to be longer (i.e., on the order of seconds or even minutes). Therefore, in order to apply iterative wavefront shaping in living biological tissue that has a decorrelation time in millisecond range, further efforts are required in hardware enhancement on top of algorithm improvement. Lastly but not the least, although no application with living tissue or fast decorrelating media has been demonstrated, this proof-of-principle study shows a concrete possibility of using a probabilitybased algorithm to achieve iterative optimization results in experiment that approach theoretical enhancement, which could add a new perspective to the current knowledge of iterative wavefront shaping. 


\section{Conclusions}

In this study, a probability-based iterative wavefront shaping algorithm is proposed that integrates ant colony optimization into a genetic algorithm for optical focusing through scattering media. In this method, a probability map is computed based on the real-time performance of the population, which is used to indicate the probability for each DMD pixel to be "ON" or "OFF" to make a positive contribution to the focusing. With clearer guidance for the new solution generation process and less involvement of randomness, we can narrow the gap between theoretical and practical performance. Experimental results approaching the theoretical optimum have been achieved with high efficiency. Besides, with the proposed algorithm, we also demonstrate higher adaptability in an unstable scattering environment and more spatially uniform optical focusing in the field of view. As a proof-of-concept study, there are a few more aspects can be further improved, such as the optimization speed. Nevertheless, the presented encouraging progress may inspire further development and applications of iterative wavefront shaping through scattering media.

\section{Methods}

The experiments are conducted with the setup shown in Fig. 3. The light from a continuous 532 nm laser (EXLSR-532-300-CDRH, Spectra Physics, USA) is expanded by convex lenses L1 and L2, so that the beam can cover all effective elements on the DMD (V-9501, Vialux, Germany) and the wavefront is iteratively modulated according to the optimization algorithms. The diameter of the modulated light from the DMD is reduced by convex lenses L3 and L4, and a $25 \mathrm{x}$ objective lens (GCO-2114 Heng Yang Guang Xue, China, NA=0.4) is used to focus the light onto a scattering medium. In our experiments, ground glass consisting of 2 pieces of $2 \mathrm{~mm}$ thick 220 grit optical diffuser (DG10-220-MD, Thorlabs, USA) and a 1-meter long multimode fiber (SUH100, Xinrui, China, diameter $=100 \mu \mathrm{m}, \mathrm{NA}=0.22$ ) are used as the scattering medium. The ground glass creates a highly stable scattering environment as it is mounted on an optical stage (M-OTS-ST-48-12-I, Newport, USA), while the multimode fiber mimics an unstable medium as the fiber body is not fixed, making it susceptible to disturbances from air flows and room vibrations. The speckle pattern behind the scattering medium is captured by a CMOS camera (Black S BFS-U3-04S2M-CS, FLIR, Canada). To ensure that the camera is well synchronized with the DMD, trigger signals are sent from the DMD to the camera so that the output light field from each input DMD pattern can be accurately captured.

\section{References}

1 Horstmeyer, R., Ruan, H. \& Yang, C. Guidestar-assisted wavefront-shaping methods for focusing light into biological tissue. Nature photonics 9, 563-571 (2015).

2 Yaqoob, Z., Psaltis, D., Feld, M. S. \& Yang, C. Optical phase conjugation for turbidity suppression in biological samples. Nature photonics 2, 110-115 (2008).

3 Papadopoulos, I. N., Farahi, S., Moser, C. \& Psaltis, D. Focusing and scanning light through a multimode optical fiber using digital phase conjugation. Optics express 20, 10583-10590 (2012).

$4 \quad$ Vellekoop, I. M., Cui, M. \& Yang, C. Digital optical phase conjugation of fluorescence in turbid tissue. Applied physics letters 101, 081108 (2012).

5 Hillman, T. R. et al. Digital optical phase conjugation for delivering two-dimensional images through turbid media. Scientific reports 3, 1-5 (2013).

6 Wang, D. et al. Focusing through dynamic tissue with millisecond digital optical phase conjugation. Optica 2, 728-735 (2015)

7 Lee, K., Lee, J., Park, J.-H., Park, J.-H. \& Park, Y. One-wave optical phase conjugation mirror by actively coupling arbitrary light fields into a single-mode reflector. Physical review letters 115, 153902 (2015)

8 Liu, Y., Ma, C., Shen, Y., Shi, J. \& Wang, L. V. Focusing light inside dynamic scattering media with millisecond digital optical phase conjugation. Optica 4, 280-288 (2017). 
Popoff, S. M. et al. Measuring the transmission matrix in optics: an approach to the study and control of light propagation in disordered media. Physical review letters 104, 100601 (2010). $\mathrm{Yu}, \mathrm{H}$. et al. Measuring large optical transmission matrices of disordered media. Physical review letters 111, 153902 (2013).

11 Chaigne, T. et al. Controlling light in scattering media non-invasively using the photoacoustic transmission matrix. Nature Photonics 8, 58-64 (2014).

Tzang, O. et al. Wavefront shaping in complex media with a $350 \mathrm{kHz}$ modulator via a 1D-to2D transform. Nature Photonics 13, 788-793 (2019).

13 Vellekoop, I. M. \& Mosk, A. Focusing coherent light through opaque strongly scattering media. Optics letters 32, 2309-2311 (2007).

14 Vellekoop, I. M. \& Mosk, A. Phase control algorithms for focusing light through turbid media. Optics communications 281, 3071-3080 (2008). optimization for focusing through turbid media in noisy environments. Optics express 20, 4840-4849 (2012). Wu, D., Luo, J., Li, Z. \& Shen, Y. A thorough study on genetic algorithms in feedback-based wavefront shaping. Journal of Innovative Optical Health Sciences 12, 1942004 (2019). Anderson, B. R., Price, P., Gunawidjaja, R. \& Eilers, H. Microgenetic optimization algorithm for optimal wavefront shaping. Applied optics 54, 1485-1491 (2015).

18 Zhang, X. \& Kner, P. Binary wavefront optimization using a genetic algorithm. Journal of Optics 16, 125704 (2014).

19 Li, B.-Q. et al. Shaping the wavefront of incident light with a strong robustness particle swarm optimization algorithm. Chinese Physics Letters 35, 124201 (2018). Hui-Ling, H., Zi-Yang, C., Cun-Zhi, S., Ji-Lin, L. \& Ji-Xiong, P. Light focusing through scattering media by particle swarm optimization. Chinese Physics Letters 32, 104202 (2015). Fang, L. et al. Binary wavefront optimization using particle swarm algorithm. Laser Physics 28, 076204 (2018).

22 Feng, Q., Zhang, B., Liu, Z., Lin, C. \& Ding, Y. Research on intelligent algorithms for amplitude optimization of wavefront shaping. Applied optics 56, 3240-3244 (2017). Woo, C. M., Li, H., Zhao, Q. \& Lai, P. Dynamic mutation enhanced particle swarm optimization for optical wavefront shaping. Optics Express 29, 18420-18426 (2021). Fang, L. et al. Binary wavefront optimization using a simulated annealing algorithm. Applied optics 57, 1744-1751 (2018). Fayyaz, Z. et al. Simulated annealing optimization in wavefront shaping controlled transmission. Applied optics 57, 6233-6242 (2018). $\mathrm{Li}, \mathrm{H}$. et al. Adaptive optical focusing through perturbed scattering media with a dynamic mutation algorithm. Photonics Research 9, 202-212 (2021).

27 Zhao, Q. et al. Parameter-free optimization algorithm for iterative wavefront shaping. Optics Letters 46, 2880-2883 (2021).

28 Wang, Z.-Q. et al. Bat algorithm-enabled binary optimization for scattered light focusing. Applied Physics Express 12, 102002 (2019). Yang, J. et al. Anti-scattering light focusing by fast wavefront shaping based on multi-pixel encoded digital-micromirror device. Light: Science \& Applications 10, 1-9 (2021). Cheng, S., Li, H., Luo, Y., Zheng, Y. \& Lai, P. Artificial intelligence-assisted light control and computational imaging through scattering media. Journal of innovative optical health sciences 12, 1930006 (2019).

31 Luo, Y., Yan, S., Li, H., Lai, P. \& Zheng, Y. Focusing light through scattering media by reinforced hybrid algorithms. APL photonics 5, 016109 (2020).

$32 \mathrm{Hu}, \mathrm{S} ., \mathrm{Hu}, \mathrm{L} ., \mathrm{Z}$, Zhang, B., Gong, W. \& Si, K. Simplifying the detection of optical distortions by machine learning. Journal of Innovative Optical Health Sciences 13, 2040001 (2020).

33 Luo, Y., Yan, S., Li, H., Lai, P. \& Zheng, Y. Towards smart optical focusing: deep learningempowered dynamic wavefront shaping through nonstationary scattering media. Photonics Research 9, B262-B278 (2021).

34 Lai, P., Wang, L., Tay, J. W. \& Wang, L. V. Photoacoustically guided wavefront shaping for enhanced optical focusing in scattering media. Nature photonics 9, 126-132 (2015).

35 Liu, Y. et al. Optical focusing deep inside dynamic scattering media with near-infrared timereversed ultrasonically encoded (TRUE) light. Nature communications 6, 1-9 (2015). 
Selvi, V. \& Umarani, R. Comparative analysis of ant colony and particle swarm optimization techniques. International Journal of Computer Applications 5, 1-6 (2010).

37 Dorigo, M. \& Di Caro, G. in Proceedings of the 1999 congress on evolutionary computationCEC99 (Cat. No. 99TH8406). 1470-1477 (IEEE).

38 Dorigo, M., Birattari, M. \& Stutzle, T. Ant colony optimization. IEEE computational intelligence magazine 1, 28-39 (2006).

39 Yang, Z., Fang, L., Zhang, X. \& Zuo, H. Controlling a scattered field output of light passing through turbid medium using an improved ant colony optimization algorithm. Optics and Lasers in Engineering 144, 106646 (2021).

40 Vellekoop, I. M. Feedback-based wavefront shaping. Optics express 23, 12189-12206 (2015).

41 Akbulut, D., Huisman, T. J., van Putten, E. G., Vos, W. L. \& Mosk, A. P. Focusing light through random photonic media by binary amplitude modulation. Optics express 19, 4017 4029 (2011).

42 Conkey, D. B. \& Piestun, R. Color image projection through a strongly scattering wall. Optics express 20, 27312-27318 (2012).

43 Bianchi, S. \& Di Leonardo, R. A multi-mode fiber probe for holographic micromanipulation and microscopy. Lab on a Chip 12, 635-639 (2012).

44 Fayyaz, Z., Mohammadian, N., Tabar, M. R. R., Manwar, R. \& Avanaki, K. A comparative study of optimization algorithms for wavefront shaping. Journal of Innovative Optical Health Sciences 12, 1942002, doi:10.1142/s1793545819420021 (2019).

45 Goodman, J. W. Statistical optics. (John Wiley \& Sons, 2015).

46 Chandrasekaran, S. N., Ligtenberg, H., Steenbergen, W. \& Vellekoop, I. M. in Emerging Digital Micromirror Device Based Systems and Applications VI. 897905 (International Society for Optics and Photonics).

47 Lu, Q.-H. et al. Quantum key distribution over scattering channel. arXiv preprint arXiv:2109.12282 (2021).

48 Li, L., Zheng, Y., Liu, H. \& Chen, X. Reconstitution of optical orbital angular momentum through strongly scattering media via feedback-based wavefront shaping method. Chinese Optics Letters 19, 100101 (2021).

\section{Acknowledgements}

The work was supported by National Natural Science Foundation of China (NSFC) (81930048, 81627805, 81671726), Hong Kong Research Grant Council (15217721, R5029-19), Hong Kong Innovation and Technology Commission (GHP/043/19SZ, GHP/044/19GD), Guangdong Science and Technology Commission (2019A1515011374, 2019BT02X105), and Hong Kong Polytechnic University (P0038180).

\section{Competing interests}

The authors declare no competing interests.

\section{Data availability}

Data underlying the results presented in this paper are available from the corresponding authors upon reasonable request.

\section{Code availability}

The codes used in this paper are available from the corresponding authors upon reasonable request.

\section{Contributions}

C.M.W., Q.Z., and Z.Y. conceived the idea; C.M.W., Q.Z., and H.L. carried out the theoretical analysis; T.Z. built the experimental setup; C.M.W., and T.Z. performed the experiments; Z.Y., and P.L. supervised the research; and C.M.W, and Z.Y. wrote the paper with contribution from all other authors. 